\title{
Simple procedures for finding mean first passage times in Markov chains
}

\author{
JEFFREY J. HUNTER \\ Institute of Information and Mathematical Sciences \\ Massey University at Albany, Auckland, New Zealand
}

The derivation of mean first passage times in Markov chains involves the solution of a family of linear equations. By exploring the solution of a related set of equations, using suitable generalized inverses of the Markovian kernel $I-P$, where $P$ is the transition matrix of a finite irreducible Markov chain, we are able to derive elegant new results for finding the mean first passage times. As a by-product we derive the stationary distribution of the Markov chain without the necessity of any further computational procedures. Standard techniques in the literature, using for example Kemeny and Snell's fundamental matrix $Z$, require the initial derivation of the stationary distribution followed by the computation of $Z$, the inverse $I-P+\boldsymbol{e} \pi^{T}$ where $\boldsymbol{e}^{T}=(1,1, \ldots, 1)$ and $\pi^{T}$ is the stationary probability vector. The procedures of this paper involve only the derivation of the inverse of a matrix of simple structure, based upon known characteristics of the Markov chain together with simple elementary vectors. No prior computations are required. Various possible families of matrices are explored leading to different related procedures.

\section{Introduction}

In solving for mean first passage times in irreducible discrete time Markov chains typically the results are expressed in terms of the elements of $Z$, Kemney and Snell's fundamental matrix, ([7]), or $A^{\#}$ the group inverse of $I-P$, (Meyer, [8]) where $P$ is the transition matrix of the Markov chain and $I$ is the identity matrix. The computation of $Z$ $=[I-P+\Pi]^{-1}$ and $A^{\#}=Z-\Pi$ both require the prior determination of $\left\{\pi_{i}\right\}$, the stationary distribution of the Markov chain. We explore the joint determination of both the stationary distribution and the mean first passage times using appropriate generalized matrix inverses that do not require previous knowledge of the stationary distribution.

In an earlier paper (Hunter [6]) the use of special classes of generalized matrix inverses was explored in order to determine expressions for the stationary probabilities and the mean first passage times, the key properties of irreducible Markov chains. In this paper we consider instead a class of generalized inverses that are in fact matrix inverses to 
give alternative expressions for the stationary probabilities and the mean first passage times. We explore the structure of these matrix inverses in order to determine if any special relationships exist to provide computational checks upon any derivations of the key properties.

\section{Generalized inverses of Markovian kernels}

Let $P=\left[p_{i j}\right]$ be the transition matrix of a finite irreducible, $m$-state Markov chain with state space $S=\{1,2, \ldots, m\}$ and stationary probability vector $\pi^{T}=\left(\pi_{1}, \pi_{2}, \ldots, \pi_{m}\right)$.

The following summary provides the key features of generalized inverses (g-inverses) of the Markovian kernel $I-P$ that we shall make use of in developing our new results. The key results below can be found in Hunter [2].

$G$ is a g-inverse, or a "Condition 1" g-inverse, of $I-P$ if and only if:

$$
(I-P) G(I-P)=I-P .
$$

Let $P$ be the transition matrix of a finite irreducible Markov chain with stationary probability vector $\pi^{T}$. Let $\boldsymbol{e}^{T}=(1,1, \ldots, 1)$ and $\boldsymbol{t}$ and $\boldsymbol{u}$ be any vectors.

(a) $I-P+\boldsymbol{t} \boldsymbol{u}^{T}$ is non-singular if and only if $\pi^{T} \boldsymbol{t} \neq 0$ and $\boldsymbol{u}^{T} \boldsymbol{e} \neq 0$.

(b) If $\pi^{T} \boldsymbol{t} \neq 0$ and $\boldsymbol{u}^{T} \boldsymbol{e} \neq 0$ then $\left[I-P+\boldsymbol{t} \boldsymbol{u}^{T}\right]^{-1}$ is a g-inverse of $I-P$.

All "Condition 1" g-inverses of $I-P$ are of the form $\left[I-P+\boldsymbol{t} \boldsymbol{u}^{T}\right]^{-1}+\boldsymbol{e} \boldsymbol{f}^{T}+\boldsymbol{g} \boldsymbol{\pi}^{T}$ for arbitrary vectors $\boldsymbol{f}$ and $g$.

G-inverses may satisfy some of the following additional conditions:

$$
\begin{array}{ll}
\text { Condition 2: } & G(I-P) G=G, \\
\text { Condition 3: } & {[(I-P) G]^{T}=(I-P) G,} \\
\text { Condition 4: } & {[G(I-P)]^{T}=G(I-P),} \\
\text { Condition 5: } & (I-P) G=(I-P) G .
\end{array}
$$

If $G$ is any g-inverse of $I-P$, define $A \equiv I-(I-P) G$ and $B \equiv I-G(I-P)$, then (Hunter [5])

$\begin{array}{ll} & G=\left[I-P+\alpha \beta^{T}\right]^{-1}+\gamma \boldsymbol{e} \pi^{T}, \\ \text { where } & \boldsymbol{\alpha}=A \boldsymbol{e}, \boldsymbol{\beta}^{T}=\pi^{T} B, \gamma+1=\pi^{T} G \boldsymbol{\alpha}=\beta^{T} G \boldsymbol{e}=\beta^{T} G \boldsymbol{\alpha} \\ \text { and } & \pi^{T} \boldsymbol{\alpha}=1, \boldsymbol{\beta}^{T} \boldsymbol{e}=1 . \\ \text { Further } & A=\boldsymbol{\alpha} \pi^{T} \\ \text { and } & B=\boldsymbol{e} \boldsymbol{\beta}^{T} .\end{array}$


The parameters $\alpha, \beta$, and $\gamma$ uniquely specify and characterize the g-inverse so that we can denote such a g-inverse as $G(\alpha, \beta, \gamma)$. In Hunter [5] it is shown that

$G(\alpha, \beta, \gamma)$ satisfies condition 2 if and only if $\gamma=-1$,

$G(\alpha, \beta, \gamma)$ satisfies condition 3 if and only if $\alpha=\pi / \pi^{T} \pi$,

$G(\alpha, \beta, \gamma)$ satisfies condition 4 if and only if $\beta=\boldsymbol{e} / \boldsymbol{e}^{T} \boldsymbol{e}$,

$G(\alpha, \beta, \gamma)$ satisfies condition 5 if and only if $\alpha=\boldsymbol{e}$ and $\beta=\pi$.

The Moore-Penrose g-inverse of $I-P$ is the unique matrix satisfying conditions $1,2,3$ and 4 and has the form $G=G\left(\pi / \pi^{T} \pi, \boldsymbol{e} / \boldsymbol{e}^{T} \boldsymbol{e},-1\right)$. (An equivalent form was originally derived by Paige, Styan and Wachter [10].)

The group inverse of $I-P$ is the (unique) $(1,2,5)$ g-inverse $A^{\#}=G(\boldsymbol{e}, \pi,-1)$, as derived by Meyer [8].

Kemeney and Snell's fundamental matrix of finite irreducible Markov chains (see [7]) is $Z=\left[I-P+\boldsymbol{e} \pi^{T}\right]^{-1}=G(\boldsymbol{e}, \pi, 0)$, a $(1,5)$ g-inverse with $\gamma=0$.

The following results are easily established (see Hunter [2])

$$
\begin{aligned}
& \boldsymbol{u}^{T}\left[I-P+\boldsymbol{t} \boldsymbol{u}^{T}\right]^{-1}=\pi^{T} /\left(\pi^{T} \boldsymbol{t}\right) . \\
& {\left[I-P+\boldsymbol{t} \boldsymbol{u}^{T}\right]^{-1} \boldsymbol{t}=\boldsymbol{e} /\left(\boldsymbol{u}^{T} \boldsymbol{e}\right) .}
\end{aligned}
$$

\section{Stationary distributions}

There are a variety of techniques that can be used for the computation of stationary distributions involving the solution of the singular system of linear equations, $\pi^{T}(I-P)$ $=\mathbf{0}^{T}$, subject to the boundary condition $\pi^{T} \boldsymbol{e}=1$.

Since, as we shall see later, the derivation of mean first passage times involves either the computation of a matrix inverse or a matrix g-inverse, we consider only those techniques for solving the stationary distributions that use g-inverses. This will assist us later to consider the joint computation of the stationary distributions and mean first passage times with a minimal set of computations.

We consider three specific classes of procedures - one using $A=I-(I-P) G$, one using $B=I-G(I-P)$, and one using simply $G$.

Theorem 3.1: ([2]) If $G$ is any g-inverse of $I-P, A \equiv I-(I-P) G$ and $v^{T}$ is any vector such that $\boldsymbol{v}^{T} A \boldsymbol{e} \neq 0$ then

$$
\pi^{T}=\frac{\boldsymbol{v}^{T} A}{\boldsymbol{v}^{T} A \boldsymbol{e}},
$$


Furthermore Ae $\neq 0$ for all g-inverse of $G$ so that it is always possible to find a suitable $v^{T}$.

Theorem 3.1 utilizes the observation that the matrix $A$ has a very special structure. From (2.4) $A=\alpha \pi^{T}$. Since, from (2.3), $\pi^{T} \alpha=1$ it is clear that $\alpha \neq \mathbf{0}$ implying $A \boldsymbol{e}=\alpha \neq 0$ and thus it is always possible to find a suitable $v^{T}$ for Theorem 3.1. Knowledge of the conditions of the g-inverse usually leads to suitable choices of $\boldsymbol{v}^{T}$ that simplify $\boldsymbol{v}^{T} A \boldsymbol{e}$.

Corollary 3.1.1: ([6]) Let $G$ be any g-inverse of $I-P$, and $A=I-(I-P) G$.

(a) For all such $G, \quad \pi^{T}=\frac{\boldsymbol{e}^{T} A^{T} A}{\boldsymbol{e}^{T} A^{T} A \boldsymbol{e}}$.

(b) If $G$ is $(1,3)$-inverse of $I-P$, and $\boldsymbol{e}_{i}^{T}$ is the $i$-th elementary vector,

$$
\pi^{T}=\frac{\boldsymbol{e}^{T} A}{\boldsymbol{e}^{T} A \boldsymbol{e}} \text { and, for any } i=1,2, \ldots, m, \pi^{T}=\frac{\boldsymbol{e}_{i}^{T} A}{\boldsymbol{e}_{i}^{T} A \boldsymbol{e}} .
$$

(c) If $G$ is $(1,5)$ g-inverse of $I-P$,

$$
\pi^{T}=\frac{\boldsymbol{e}^{T} A}{\boldsymbol{e}^{T} \boldsymbol{e}} \text { and, for any } i=1,2, \ldots, m, \pi^{T}=\boldsymbol{e}_{i}^{T} A
$$

In certain cases the expression $B=I-G(I-P)$ can also be used to find an expression for $\pi^{T}$.

Theorem 3.2: ([6]) Let $G$ be any g-inverse of $I-P$ that is not a $(1,2) g$-inverse, $B=$ $I-G(I-P)$ and $\boldsymbol{v}^{T}$ any vector such that $\boldsymbol{v}^{T} \boldsymbol{e} \neq 0$. Then

$$
\pi^{T}=\frac{\boldsymbol{v}^{T} B G}{\boldsymbol{v}^{T} B G \boldsymbol{e}}
$$

Corollary 3.2.1: ([6]) Let $G$ be any g-inverse of $I-P$, and $B=I-G(I-P)$.

(a) For all $G$, except a $(1,2)$ g-inverse,

$$
\pi^{T}=\frac{\boldsymbol{e}^{T} B G}{\boldsymbol{e}^{T} B G \boldsymbol{e}} \text { and }, \text { for any } i=1,2, \ldots, m, \pi^{T}=\frac{\boldsymbol{e}_{i}^{T} B G}{\boldsymbol{e}_{i}^{T} B G \boldsymbol{e}} .
$$

(b) If $G$ is a $(1,5)$ g-inverse of $I-P$, then for any $i=1,2, \ldots, m, \pi^{T}=\boldsymbol{e}_{i}^{T} B$.

The above theorems and corollaries all require computation of $A$ or $B$, based upon prior knowledge of $G$. If $G$ is of special structure one can often find an expression for $\pi^{T}$ in terms of $G$ alone.

Theorem 3.3: ([6]) If G is a $(1,4)$ g-inverse of $I-P, \pi^{T}=\frac{\boldsymbol{e}^{T} G}{\boldsymbol{e}^{T} G \boldsymbol{e}}$. 
Some of the above expressions are well known. Theorem 3.1 appears in Hunter [2], [3]. The first expression of Corollary 3.1.1 (b) was originally derived by Decell and Odell [1]. Meyer [8] established the first expression of Corollary 3.1.1 (c) under the assumption that $G$ is a $(1,2,5)$ g-inverse (but the 2-condition is not necessary).

If $\boldsymbol{v}^{T}=\boldsymbol{e}_{i}^{T}$, the $i$-th elementary vector, then $\boldsymbol{e}_{i}^{T} A \boldsymbol{e}=\boldsymbol{e}_{i}^{T} \boldsymbol{\alpha}=\alpha_{i}$, which must be non-zero for at least one such $i$. Since $\boldsymbol{e}_{i}^{T} A$ consists of elements of the $i$-th row of $A$, we can always find at least one row of $A$ that does not contain a non-zero element. Furthermore, if there is at least one non-zero element in that row, all the elements in that row must be non-zero, since the rows of $A$ are scaled versions of $\pi^{T}$. Thus, if $A=\left[a_{i j}\right]$ then there is at least one $i$ such $a_{i 1} \neq 0$ in which case $a_{i j} \neq 0$ for $j=1, \ldots, m$. This leads to following result.

Theorem 3.4: ([6]) Let $G$ be any g-inverse of $I-P$. Let $A=I-(I-P) G \equiv\left[a_{i j}\right]$.

Let $r$ be the smallest integer $i(1 \leq i \leq m)$ such that $\sum_{k=1}^{m} a_{i k} \neq 0$, then

$$
\pi_{j}=\frac{a_{r j}}{\sum_{k=1}^{m} a_{r k}}, j=1,2, \ldots, m .
$$

In applying Theorem 3.4 one typically needs to first find $a_{11}\left(=1-g_{11}+\sum_{k=1}^{m} p_{1 k} g_{k 1}\right)$. If $a_{11} \neq 0$ then the first row of $A$ will suffice to find the stationary probabilities. If not find $a_{21}, a_{31}, \ldots$ and stop at the first non-zero $a_{r 1}$.

For some specific g-inverses we need only find the first row of $A$. For example MATLAB uses the pseudo inverse routine $\operatorname{pinv}(I-P)$, to generate the $(1,2,3,4) \mathrm{g}$ inverse of $I-P$.

Corollary 3.4.1: ( $[6])$ If $G$ is a $(1,3)$ or $(1,5) g$-inverse of $I-P$, and if $A=I-(I-$ $P) G \equiv\left[a_{i j}\right]$ then

$$
\pi_{j}=\frac{a_{1 j}}{\sum_{k=1}^{m} a_{1 k}}, j=1,2, \ldots, m .
$$

Proof: If $G$ satisfies condition $3, \alpha=\pi / \pi^{T} \pi$ in which case $\alpha_{1} \neq 0$. Similarly if $G$ satisfies condition 5, $\alpha=\boldsymbol{e}$ in which case $\alpha_{1}=1$. The non-zero form of $\alpha_{1}$ ensures $a_{11} \neq 0$.

G-inverse conditions 2 or 4 do not place any restrictions upon $\alpha$ and consequently the non-zero nature of $a_{11}$ cannot be guaranteed in these situations. 
While (3.1), (3.2) and (3.3) are useful expressions for obtaining the stationary probabilities, the added computation of $A$ following the derivation of a g-inverse $G$ is typically unnecessary, especially when additional special properties of $G$ are given.

Rather than classifying $G$ as a specific "multi-condition" g-inverse, we now focus on special class of g-inverses which are matrix inverses of the simple form $\left[I-P+\boldsymbol{t} \boldsymbol{u}^{T}\right]^{-1}$, where $\boldsymbol{t}$ and $\boldsymbol{u}^{\boldsymbol{T}}$ are simple forms, selected to ensure that the inverse exists with $\pi^{T} \boldsymbol{t} \neq 0$ and $\boldsymbol{u}^{T} \boldsymbol{e} \neq 0$. A general result for deriving an expression for $\pi^{T}$ using such a g-inverse is the following.

Theorem 3.5: If $G=\left[I-P+\boldsymbol{t u}^{T}\right]^{-1}$ where $\boldsymbol{u}$ and $\boldsymbol{t}$ are any vectors such that $\pi^{T} \boldsymbol{t} \neq 0$ and $\boldsymbol{u}^{T} \boldsymbol{e} \neq 0$, then

$$
\pi^{T}=\frac{\boldsymbol{u}^{T} G}{\boldsymbol{u}^{T} G \boldsymbol{e}} .
$$

Hence, if $G=\left[g_{i j}\right]$ and $\boldsymbol{u}^{T}=\left(u_{1}, u_{2}, \ldots, u_{m}\right)$,

$$
\pi_{j}=\frac{\sum_{k=1}^{m} u_{k} g_{k j}}{\sum_{r=1}^{m} u_{r} \sum_{s=1}^{m} g_{r s}}=\frac{\sum_{k=1}^{m} u_{k} g_{k j}}{\sum_{r=1}^{m} u_{r} g_{r} .}, j=1,2, \ldots, m .
$$

Proof: Using (2.6) it is easily seen that $\boldsymbol{u}^{T}\left[I-P+\boldsymbol{t} \boldsymbol{u}^{T}\right]^{-1} \boldsymbol{e}=\pi^{T} \boldsymbol{e} / \pi^{T} \boldsymbol{t}=1 / \boldsymbol{\pi}^{T} \boldsymbol{t}$ and (3.4) follows. The elemental expression (3.5) follows from (3.4).

The form for $\pi^{T}$ above has the added simplification that we need only determine $G$ (and not $A$ or $B$ as in Theorems 3.1 and 3.2 and their corollaries.) While it will be necessary to evaluate the inverse of the matrix $I-P+\boldsymbol{t} \boldsymbol{u}^{T}$ this may either be the inverse of a matrix which has a simple special structure or the inverse itself may be one that has a simple structure. Further, we also wish to use this inverse to assist in the determination of the mean first passage times (see Section 4).

We consider special choices of $\boldsymbol{t}$ and $\boldsymbol{u}$ based either upon the simple elementary vectors $\boldsymbol{e}_{i}$, the unit vector $\boldsymbol{e}$, the rows and/or columns of the transition matrix $P$, and in one case a combination of such elements. Let $\boldsymbol{p}_{a}^{(c)} \equiv P \boldsymbol{e}_{a}$ denote the $a$-th column of $P$ and $\boldsymbol{p}_{b}^{(r) T} \equiv \boldsymbol{e}_{b}^{T} P$ denote the $b$-th row of $P$.

Table 1 below lists of a variety of special g-inverses with their specific parameters. All these results follow from the observation that if $G=\left[I-P+\boldsymbol{t} \boldsymbol{u}^{T}\right]^{-1}$ then, from (2.2), the parameters are given by $\boldsymbol{\alpha}=\boldsymbol{t} / \boldsymbol{\pi}^{T} \boldsymbol{t}, \boldsymbol{\beta}=\boldsymbol{u}^{T} / \boldsymbol{u}^{T} \mathbf{e}$ and $\gamma+1=1 /\left\{\left(\pi^{T} \boldsymbol{t}\right)\left(\boldsymbol{u}^{T} \boldsymbol{e}\right)\right\}$. The special structure of the g-inverses given in Table 1 leads, in many cases, to very simple forms for the stationary probabilities. 
In applying Theorem 3.5, observe that $\pi^{T}=\boldsymbol{u}^{T} G$ if and only if $\boldsymbol{u}^{T} G \boldsymbol{e}=1$ if and only if $\pi^{T} \boldsymbol{t}=1$.

Table 1: Special g-inverses

\begin{tabular}{|c|l|l|l|l|}
\hline Identifier & \multicolumn{1}{|c|}{ g-inverse } & \multicolumn{3}{|c|}{ Parameters } \\
\hline & {$\left[I-P+\boldsymbol{t u}^{T}\right]^{-1}$} & $\boldsymbol{\alpha}$ & $\boldsymbol{\beta}^{T}$ & $\gamma$ \\
\hline$G_{e e}$ & {$\left[I-P+\boldsymbol{e} \boldsymbol{e}^{T}\right]^{-1}$} & $\boldsymbol{e}$ & $\boldsymbol{e}^{T} / m$ & $(1 / m)-1$ \\
\hline$G_{e b}^{(r)}$ & {$\left[I-P+\boldsymbol{e} \boldsymbol{p}_{b}^{(r) T}\right]^{-1}$} & $\boldsymbol{e}$ & $\boldsymbol{p}_{b}^{(r) T}$ & 0 \\
\hline$G_{e b}$ & {$\left[I-P+\boldsymbol{e} \boldsymbol{e}_{b}^{T}\right]^{-1}$} & $\boldsymbol{e}$ & $\boldsymbol{e}_{b}^{T}$ & 0 \\
\hline$G_{a e}^{(c)}$ & {$\left[I-P+\boldsymbol{p}_{a}^{(c)} \boldsymbol{e}^{T}\right]^{-1}$} & $\boldsymbol{p}_{a}^{(c)} / \pi_{a}$ & $\boldsymbol{e}^{T} / m$ & $\left(1 / m \pi_{a}\right)-1$ \\
\hline$G_{a b}^{(c, r)}$ & {$\left[I-P+\boldsymbol{p}_{a}^{(c)} \boldsymbol{p}_{b}^{(r) T}\right]^{-1}$} & $\boldsymbol{p}_{a}^{(c)} / \pi_{a}$ & $\boldsymbol{p}_{b}^{(r) T}$ & $\left(1 / \pi_{a}\right)-1$ \\
\hline$G_{a b}^{(c)}$ & {$\left[I-P+\boldsymbol{p}_{a}^{(c)} \boldsymbol{e}_{b}^{T}\right]^{-1}$} & $\boldsymbol{p}_{a}^{(c)} / \pi_{a}$ & $\boldsymbol{e}_{b}^{T}$ & $\left(1 / \pi_{a}\right)-1$ \\
\hline$G_{a e}$ & {$\left[I-P+\boldsymbol{e}_{a} \boldsymbol{e}^{T}\right]^{-1}$} & $\boldsymbol{e}_{a} / \pi_{a}$ & $\boldsymbol{e}^{T} / m$ & $\left(1 / m \pi_{a}\right)-1$ \\
\hline$G_{a b}^{(r)}$ & {$\left[I-P+\boldsymbol{e}_{a} \boldsymbol{p}_{b}^{(r) T}\right]^{-1}$} & $\boldsymbol{e}_{a} / \pi_{a}$ & $\boldsymbol{p}_{b}^{(r) T}$ & $\left(1 / \pi_{a}\right)-1$ \\
\hline$G_{a b}$ & {$\left[I-P+\boldsymbol{e}_{a} \boldsymbol{e}_{b}^{T}\right]^{-1}$} & $\boldsymbol{e}_{a} / \pi_{a}$ & $\boldsymbol{e}_{b}^{T}$ & $\left(1 / \pi_{a}\right)-1$ \\
\hline$G_{t b}^{(c)}$ & {$\left[I-P+\boldsymbol{t}_{b} \boldsymbol{e}_{b}^{T}\right]^{-1}$} & $\boldsymbol{t}_{b}$ & $\boldsymbol{e}_{b}^{T}$ & 0 \\
& $\left(\boldsymbol{t}_{b} \equiv \boldsymbol{e}-\boldsymbol{e}_{b}+\boldsymbol{p}_{b}^{(c)}\right)$ & & & \\
\hline
\end{tabular}

Simple sufficient conditions for $\pi^{T} \boldsymbol{t}=1$ are $\boldsymbol{t}=\boldsymbol{e}$ or $\boldsymbol{t}=\alpha$ (cf. (2.3)). (This later condition is of use only if $\alpha$ does not explicitly involve any of the stationary probabilities, as for $G_{t b}^{(c)}$ )

$G_{t b}^{(c)}$ is included in Table 1 as the update $\boldsymbol{t}_{b} \boldsymbol{e}_{b}^{T}$ replaces the $b$-th column of $I-P$ by $\boldsymbol{e}$. (See [10]).

Corollary 3.5.1: If $G=\left[I-P+\boldsymbol{e u}^{T}\right]^{-1}$ where $\boldsymbol{u}^{T} \boldsymbol{e} \neq 0$,

$$
\pi^{T}=\boldsymbol{u}^{T} G \text {. }
$$

and hence if $\boldsymbol{u}^{T}=\left(u_{1}, u_{2}, \ldots, u_{m}\right)$ and $G=\left[g_{i j}\right]$ then

$$
\pi_{j}=\sum_{k=1}^{m} u_{k} g_{k j}, j=1,2, \ldots, m .
$$

In particular, we have the following special cases:

(a) If $\boldsymbol{u}^{T}=\boldsymbol{e}^{T}$ then $G \equiv G_{\boldsymbol{e} e}=\left[I-P+\boldsymbol{e} \boldsymbol{e}^{T}\right]^{-1}=\left[g_{i j}\right]$ and

$$
\pi_{j}=\sum_{k=1}^{m} g_{k j} \equiv g_{. j} .
$$


(b) If $\boldsymbol{u}^{T}=\boldsymbol{p}_{b}^{(r) T}$ then $G \equiv G_{e b}^{(r)}=\left[I-P+\boldsymbol{e p}_{b}^{(r) T}\right]^{-1}=\left[g_{i j}\right]$ and

$$
\pi_{j}=\sum_{k=1}^{m} p_{b k} g_{k j} .
$$

(c) If $\boldsymbol{u}^{T}=\boldsymbol{e}_{b}^{T}$ then $G \equiv G_{e b}=\left[I-P+\boldsymbol{e e}_{b}^{T}\right]^{-1}=\left[g_{i j}\right]$ and

$$
\pi_{j}=g_{b j} \text {. }
$$

Corollary 3.5.2: If $G=\left[I-P+\boldsymbol{t} \boldsymbol{e}^{T}\right]^{-1}$ where $\pi^{T} \boldsymbol{t} \neq 0$,

$$
\pi^{T}=\frac{\boldsymbol{e}^{T} G}{\boldsymbol{e}^{T} G \boldsymbol{e}}
$$

and hence, if $G=\left[g_{i j}\right]$, then

$$
\pi_{j}=\frac{\sum_{k=1}^{m} g_{k j}}{\sum_{r=1}^{m} \sum_{s=1}^{m} g_{r s}}=\frac{g_{. j}}{g_{. .}}, j=1,2, \ldots, m .
$$

In particular, results (3.12) hold for $G=G_{a e}^{(c)}, G_{e e}$ and $G_{a e}$.

In the special case of $G_{e e}$, using (2.6) or (2.7), it follows that $g_{. .}=1$, and (3.12) reduces to $(3.8)$.

Corollary 3.5.3: If $G=\left[I-P+\boldsymbol{t} \boldsymbol{e}_{b}^{T}\right]^{-1}$ where $\pi^{T} \boldsymbol{t} \neq 0$,

$$
\pi^{T}=\frac{\boldsymbol{e}_{b}^{T} G}{\boldsymbol{e}_{b}^{T} G \boldsymbol{e}}
$$

and hence, if $G=\left[g_{i j}\right]$, then

$$
\pi_{j}=\frac{g_{b j}}{\sum_{s=1}^{m} g_{b s}}=\frac{g_{b j}}{g_{b .}}, j=1,2, \ldots, m .
$$

In particular, results (3.14) hold for $G=G_{a b}^{(c)}, G_{a b}, G_{e b}$ and $G_{t b}^{(c)}$.

In the special cases of $G_{e b}$ and $G_{t b}^{(c)}, g_{b}=1$ and (3.14) reduces to (3.10).

Corollary 3.5.4: If $G=\left[I-P+\boldsymbol{t p}_{b}^{(r) T}\right]^{-1}$ where $\boldsymbol{\pi}^{T} \boldsymbol{t} \neq 0$,

$$
\pi^{T}=\frac{\boldsymbol{p}_{b}^{(r) T} G}{\boldsymbol{p}_{b}^{(r) T} G \boldsymbol{e}}
$$

and hence, if $G=\left[g_{i j}\right]$, then 


$$
\pi_{j}=\frac{\sum_{k=1}^{m} p_{b k} g_{k j}}{\sum_{i=1}^{m} \sum_{s=1}^{m} p_{b i} g_{i s}}, j=1,2, \ldots, m
$$

In particular, results (3.16) hold for $G=G_{a b}^{(c, r)}, G_{a b}^{(r)}$ and $G_{e b}^{(r)}$.

In the special case of $G_{e b}^{(r)}$, the denominator of (3.16) is 1 and (3.16) reduces to (3.9).

Thus we have been able to find simple elemental expressions for the stationary probabilities using any of the g-inverses in Table 1 . In the special cases of $G_{e e}, G_{e b}^{(r)}$, $G_{e b}$ and $G_{t b}^{(c)}$ the denominator of the expression given by equations (3.5) is always 1. (In each other case, observe that denominator of the expression $\boldsymbol{u}^{T} G \boldsymbol{e}$ is in fact $1 / \pi_{b}$, with $\left.\boldsymbol{u}^{T} G=\pi^{T} / \pi_{b}.\right)$

We consider the g-inverses of Table 1 in more detail in order to highlight their structure or special properties that may provide either a computational check or a reduction in the number of computations required.

Let $\boldsymbol{g}_{a}^{(c)}=G \boldsymbol{e}_{a}$ denote the $a$-th column of $G$ and $\boldsymbol{g}_{b}^{(r) T}=\boldsymbol{e}_{b}^{T} G$ denote the $b$-th row of $G$. From the definition of $G=\left[I-P+\boldsymbol{t u}^{T}\right]^{-1}$, pre- and post-multiplication by $I-P+\boldsymbol{t} \boldsymbol{u}^{T}$ yields

$$
\begin{aligned}
& G-P G+\boldsymbol{t} \boldsymbol{u}^{T} G=I, \\
& G-G P+G \boldsymbol{t} \boldsymbol{u}^{T}=I .
\end{aligned}
$$

Pre-multiplication by $\pi^{T}$ and post-multiplication by $\boldsymbol{e}$ yields the expressions given by (2.6) and (2.7), i.e. $\boldsymbol{u}^{T} G=\pi^{T} / \pi^{T} \boldsymbol{t}$ and $G \boldsymbol{t}=\boldsymbol{e} / \boldsymbol{u}^{T} \boldsymbol{e}$. Relationships between the rows, columns and elements of $G$ follow from (3.17) and (3.18) by pre- and postmultiplication by $\boldsymbol{e}_{a}$ and $\boldsymbol{e}_{b}^{T}$ and the fact that $g_{i .}=\boldsymbol{g}_{i}^{(r) T} \boldsymbol{e}, g_{. j}=\boldsymbol{e}^{T} \boldsymbol{g}_{j}^{(c)}, g_{i j}=\boldsymbol{e}_{i}^{T} G \boldsymbol{e}_{j}$. These are summarised in the following theorem.

Theorem 3.6: For any $g$ - inverse of the form $G=\left[I-P+\boldsymbol{t} \boldsymbol{u}^{T}\right]^{-1}$, with $\pi^{T} \boldsymbol{t} \neq 0$ and $u^{T} e \neq 0$,
(a) (Row properties)

$$
\begin{aligned}
& \boldsymbol{g}_{i}^{(r) T}-\boldsymbol{p}_{i}^{(r) T} G=\boldsymbol{e}_{i}^{T}-\left(t_{i} / \pi^{T} \boldsymbol{t}\right) \pi^{T} \\
& \boldsymbol{g}_{i}^{(r) T}-\boldsymbol{g}_{i}^{(r) T} P=\boldsymbol{e}_{i}^{T}-\left(1 / \boldsymbol{u}^{T} \boldsymbol{e}\right) \boldsymbol{u}^{T}
\end{aligned}
$$
and hence

$$
g_{i .}=\sum_{k=1}^{m} p_{i k} g_{k .}+1+t_{i} /\left(\sum_{k=1}^{m} \pi_{k} t_{k}\right) \text {. }
$$
(b) (Column properties)

$$
\boldsymbol{g}_{j}^{(c)}-P \boldsymbol{g}_{i}^{(c)}=\boldsymbol{e}_{j}-\left(\pi_{j} / \pi^{T} \boldsymbol{t}\right) \boldsymbol{t},
$$




$$
\begin{aligned}
& \boldsymbol{g}_{j}^{(c)}-G \boldsymbol{p}_{i}^{(c)}=\boldsymbol{e}_{j}-\left(u_{j} / \boldsymbol{u}^{T} \boldsymbol{e}\right) \boldsymbol{e}, \\
\text { and hence } & g_{. j}=\sum_{k=1}^{m} p_{. k} g_{k j}+1+\pi_{j}\left(\sum_{k=1}^{m} t_{k}\right) /\left(\sum_{k=1}^{m} \pi_{k} t_{k}\right), \\
g_{. j} & =\sum_{k=1}^{m} g_{. k} p_{k j}+1+m u_{j} /\left(\sum_{k=1}^{m} u_{k}\right) . \\
\text { (c) (Element properties) } \quad g_{i j} & =\sum_{k=1}^{m} p_{i k} g_{k j}+\delta_{i j}-t_{i} \pi_{j} /\left(\sum_{k=1}^{m} \pi_{k} t_{k}\right), \\
g_{i j} & =\sum_{k=1}^{m} g_{i k} p_{k j}+\delta_{i j}-u_{j} /\left(\sum_{k=1}^{m} u_{k}\right) .
\end{aligned}
$$

Let $\boldsymbol{g}_{\text {rowsum }}=G \boldsymbol{e}=\sum_{j=1}^{m} \boldsymbol{g}_{j}^{(c)}=\left[g_{1}, g_{2 .}, \ldots, g_{m .}\right]^{T}$ denote the column vector of row sums of $G$ and $\boldsymbol{g}_{\text {colsum }}^{T}=\boldsymbol{e}^{T} G=\sum_{j=1}^{m} \boldsymbol{g}_{j}^{(r) T}=\left[g_{.1}, g_{.2}, \ldots, g_{. m}\right]$ the row vector of column sums of $G$.

Table 2 is constructed using results of (2.6), (2.7), Theorem 3.6 and the requisite definitions.

A key observation is that stationary distribution can be found in terms of just the elements of the $b$-th row of $G_{e b}, G_{a b}^{(c)}, G_{a b}^{(r)}(a \neq b), G_{a b}$ and $G_{t b}^{(c)}$. This requires the determination of just $m$ elements of $G$. We exploit these particular matrices later.

If the entire g-inverse has been computed the stationary distribution can be found in terms of $\boldsymbol{g}_{\text {colsum }}^{T}$, the row vector of column sums, in the case of $G_{e e}, G_{a e}^{(c)}$ and $G_{a e}$. In each of these cases there are simple constraints on $\boldsymbol{g}_{a}^{(c)}$ and $\boldsymbol{g}_{\text {rowsum }}$, possibly reducing the number of computations required, or at least providing a computational check.

In the remaining cases of $G_{e b}^{(r)}, G_{a b}^{(c, r)}$ and $G_{a b}^{(r)}$, the additional computation of $\boldsymbol{p}_{b}^{(r) T} G$ is required to lead to an expression for the stationary probabilities.

We can further explore inter-relationships between some of the g-inverses in Table 1 by utilizing the following result given by Theorem 3.3 of Hunter [4].

Theorem 3.7: Let $P$ be the transition matrix of a finite irreducible transition matrix of a Markov chain with stationary probability vector $\pi^{T}$. Suppose that for $i=1,2, \pi^{T} \boldsymbol{t}_{i} \neq 0$ and $\boldsymbol{u}_{i}^{T} \boldsymbol{e} \neq 0$. Then

and hence that

$$
\left[I-P+\boldsymbol{t}_{2} \boldsymbol{u}_{2}^{T}\right]^{-1}=\left[I-\frac{\boldsymbol{e} \boldsymbol{u}_{2}^{T}}{\boldsymbol{u}_{2}^{T} \boldsymbol{e}}\right]\left[I-P+\boldsymbol{t}_{1} \boldsymbol{u}_{1}^{T}\right]^{-1}\left[I-\frac{\boldsymbol{t}_{2} \pi^{T}}{\pi^{T} \boldsymbol{t}_{2}}\right]+\frac{\boldsymbol{e} \pi^{T}}{\left(\pi^{T} \boldsymbol{t}_{2}\right)\left(\boldsymbol{u}_{2}^{T} \boldsymbol{e}\right)} .
$$




$$
\begin{aligned}
& {\left[I-P+\boldsymbol{t}_{2} \boldsymbol{u}_{2}^{T}\right]^{-1}-\left[I-P+\boldsymbol{t}_{1} \boldsymbol{u}_{1}^{T}\right]^{-1}} \\
& =\frac{\boldsymbol{e} \boldsymbol{u}_{2}^{T}}{\boldsymbol{u}_{2}^{T} \boldsymbol{e}}\left[I-P+\boldsymbol{t}_{1} \boldsymbol{u}_{1}^{T}\right]^{-1} \frac{\boldsymbol{t}_{2} \pi^{T}}{\pi^{T} \boldsymbol{t}_{2}}-\frac{\boldsymbol{e} \boldsymbol{u}_{2}^{T}}{\boldsymbol{u}_{2}^{T} \boldsymbol{e}}\left[I-P+\boldsymbol{t}_{1} \boldsymbol{u}_{1}^{T}\right]^{-1}-\left[I-P+\boldsymbol{t}_{1} \boldsymbol{u}_{1}^{T}\right]^{-1} \frac{\boldsymbol{t}_{2} \pi^{T}}{\pi^{T} \boldsymbol{t}_{2}}+\frac{\boldsymbol{e} \pi^{T}}{\left(\pi^{T} \boldsymbol{t}_{2}\right)\left(\boldsymbol{u}_{2}^{T} \boldsymbol{e}\right)} .
\end{aligned}
$$

In particular, we wish to focus on the differences between $G_{a a}^{(c)}, G_{a a}^{(r)}$ and $G_{a a}$. These results are used in Section 4.

\begin{tabular}{|c|c|c|c|c|c|c|c|}
\hline $\begin{array}{c}G \\
\text { g-inverse }\end{array}$ & $t$ & $u^{T}$ & $\begin{array}{c}\boldsymbol{g}_{a}^{(c)} \\
\text { a-th column }\end{array}$ & $\begin{array}{c}\boldsymbol{g}_{\text {colsum }}^{T} \\
\text { Column } \\
\text { sum }\end{array}$ & $\begin{array}{c}\boldsymbol{g}_{b}^{(r) T} \\
b \text {-th row }\end{array}$ & $\begin{array}{l}\boldsymbol{g}_{\text {rowsum }} \\
\text { Row } \\
\text { sum }\end{array}$ & Other properties \\
\hline$G_{e e}$ & $e$ & $\boldsymbol{e}^{T}$ & & $\pi^{T}$ & & $\boldsymbol{e} / \mathrm{m}$ & \\
\hline$G_{e b}^{(r)}$ & $e$ & $\boldsymbol{p}_{b}^{(r) T}$ & & & $\boldsymbol{e}_{b}^{T}$ & $e$ & $\boldsymbol{p}_{b}^{(r) T} G=\pi^{T}$ \\
\hline$G_{e b}$ & $e$ & $\boldsymbol{e}_{b}^{T}$ & & & $\pi^{T}$ & $e$ & \\
\hline$G_{a e}^{(c)}$ & $\boldsymbol{p}_{a}^{(c)}$ & $e^{T}$ & $e_{a}$ & $\pi^{T} / \pi_{a}$ & & & $G \boldsymbol{p}_{a}^{(c)}=\boldsymbol{e} / m$ \\
\hline$G_{a b}^{(c, r)}$ & $\boldsymbol{p}_{a}^{(c)}$ & $\boldsymbol{p}_{b}^{(r) T}$ & $\boldsymbol{e}_{a}+\left(1-p_{b a}\right) \boldsymbol{e}$ & & & & $\begin{array}{l}\boldsymbol{p}_{b}^{(r) T} G=\pi^{T} / \pi_{a} \\
G \boldsymbol{p}_{a}^{(c)}=\boldsymbol{e}\end{array}$ \\
\hline$G_{a a}^{(c)}(a=b)$ & $\boldsymbol{p}_{a}^{(c)}$ & $\boldsymbol{e}_{b}^{T}$ & $\boldsymbol{e}_{a}$ & & $\pi^{T} / \pi_{a}$ & & \\
\hline$G_{a b}^{(c)}(a \neq b)$ & $p_{a}^{(c)}$ & $\boldsymbol{e}_{b}^{T}$ & $\boldsymbol{e}+\boldsymbol{e}_{a}$ & & $\pi^{T} / \pi_{a}$ & & \\
\hline$G_{a e}$ & $\boldsymbol{e}_{a}$ & $e^{T}$ & $\boldsymbol{e} / \mathrm{m}$ & $\pi^{T} / \pi_{a}$ & & & \\
\hline$G_{a a}^{(r)}(a=b)$ & $e_{a}$ & $\boldsymbol{p}_{b}^{(r) T}$ & $e$ & & $\boldsymbol{e}_{a}^{T}$ & & $\boldsymbol{p}_{b}^{(r) T} G=\pi^{T} / \pi_{b}$ \\
\hline$G_{a b}^{(r)}(a \neq b)$ & $\boldsymbol{e}_{a}$ & $\boldsymbol{p}_{b}^{(r) T}$ & $e$ & & $\boldsymbol{e}_{b}^{T}+\pi^{T} / \pi$ & & $\boldsymbol{p}_{b}^{(r) T} G=\pi^{T} / \pi_{a}$ \\
\hline$G_{a b}$ & $\boldsymbol{e}_{a}$ & $\boldsymbol{e}_{b}^{T}$ & $e$ & & $\pi^{T} / \pi_{a}$ & & \\
\hline$G_{t b}^{(c)}$ & $t_{b}$ & $\boldsymbol{e}_{b}^{T}$ & & & $\pi^{T}$ & $\boldsymbol{e}_{b}$ & $G \boldsymbol{t}_{b}=\boldsymbol{e}$ \\
\hline
\end{tabular}

Table 2: Row and column properties of g-inverses 
Theorem 3.8:

$$
\begin{aligned}
& G_{a a}^{(c)}-G_{a a}^{(r)}=\frac{\boldsymbol{e}_{a} \pi^{T}}{\pi_{a}}-\boldsymbol{e} \boldsymbol{e}_{a}^{T} \\
& G_{a a}-G_{a a}^{(r)}=\frac{\boldsymbol{e} \pi^{T}}{\pi_{a}}-\boldsymbol{e} \boldsymbol{e}_{a}^{T}=\boldsymbol{e}\left(\frac{\pi^{T}}{\pi_{a}}-\boldsymbol{e}_{a}^{T}\right) . \\
& G_{a a}-G_{a a}^{(c)}=\frac{\boldsymbol{e} \pi^{T}}{\pi_{a}}-\frac{\boldsymbol{e}_{a} \pi^{T}}{\pi_{a}}=\left(\boldsymbol{e}-\boldsymbol{e}_{a}\right) \frac{\pi^{T}}{\pi_{a}} .
\end{aligned}
$$

\section{Proof:}

(a) Using the results of Theorem 3.7, it is easily seen that

$G_{a a}^{(c)}-G_{a a}^{(r)}=\frac{\boldsymbol{e} \boldsymbol{e}_{a}^{T}}{\boldsymbol{e}_{a}^{T} \boldsymbol{e}} G_{a a}^{(r)} \frac{\boldsymbol{p}_{a}^{(c)} \pi^{T}}{\pi^{T} \boldsymbol{p}_{a}^{(c)}}-\frac{\boldsymbol{e} \boldsymbol{e}_{a}^{T}}{\boldsymbol{e}_{a}^{T} \boldsymbol{e}} G_{a a}^{(r)}-G_{a a}^{(r)} \frac{\boldsymbol{p}_{a}^{(c)} \pi^{T}}{\pi^{T} \boldsymbol{p}_{a}^{(c)}}+\frac{\boldsymbol{e} \pi^{T}}{\left(\boldsymbol{e}_{a}^{T} \boldsymbol{e}\right)\left(\pi^{T} \boldsymbol{p}_{a}^{(c)}\right)}$.

Using, $\boldsymbol{e}_{a}^{T} \boldsymbol{e}=1, \boldsymbol{e}_{a}^{T} G_{a a}^{(r)}=\boldsymbol{e}_{a}^{T}, \boldsymbol{p}_{a}^{(c)}=P \boldsymbol{e}_{a}, \pi^{T} \boldsymbol{p}_{a}^{(c)}=\pi_{a}, \boldsymbol{e}_{a}^{T} P \boldsymbol{e}_{a}=p_{a a}$,

equation (3.22) simplifies to

$$
G_{a a}^{(c)}-G_{a a}^{(r)}=\frac{p_{a a} \boldsymbol{e} \pi^{T}}{\pi_{a}}-\boldsymbol{e} \boldsymbol{e}_{a}^{T}-\frac{G_{a a}^{(r)} P \boldsymbol{e}_{\mathrm{a}} \pi^{T}}{\pi_{a}}+\frac{\boldsymbol{e} \pi^{T}}{\pi_{a}} .
$$

Now observe that, by the definition of $G_{a a}^{(r)}$,

$$
I=G_{a a}^{(r)}-G_{a a}^{(r)} P+G_{a a}^{(r)} \boldsymbol{e}_{a} p_{a}^{(r) T} .
$$

Post-multiplying (3.33) by $\boldsymbol{e}_{a}$ yields

$$
\boldsymbol{e}_{a}=G_{a a}^{(r)} \boldsymbol{e}_{a}-G_{r r}^{(r)} P \boldsymbol{e}_{a}+G_{a a}^{(r)} \boldsymbol{e}_{a} \boldsymbol{e}_{a}^{T} P \boldsymbol{e}_{a}=\boldsymbol{e}-G_{a a}^{(r)} P \boldsymbol{e}_{a}+\boldsymbol{e} p_{a a} .
$$

Substitution of the expression for $G_{a a}^{(r)} P \boldsymbol{e}_{a}$ from (3.25) into (3.23) yields (3.19).

(b) and (c) These results follow directly from Theorem 3.7 and the row and column properties of $G_{a a}^{(r)}$ and $G_{a a}^{(c)}$, as given in Table 2 .

A close study of equation (3.19) shows that $G_{a a}^{(c)}$ and $G_{a a}^{(r)}$ differ only in the $a$-th row and $a$-th column, with specific elements in the $a$-th row and column in each matrix as given in Table 2, and with all the other elements identical. A formal proof follows from (3.29), since for $i \neq a$ and $j \neq a$, the $(i, j)$-th element of $G_{a a}^{(c)}-G_{a a}^{(r)}$ is given by

$$
\boldsymbol{e}_{i}^{T}\left(G_{a a}^{(c)}-G_{a a}^{(r)}\right) \boldsymbol{e}_{j}=\left(\boldsymbol{e}_{i}^{T} \boldsymbol{e}_{a}\right)\left(\frac{\pi^{T} \boldsymbol{e}_{j}}{\pi_{a}}\right)-\left(\boldsymbol{e}_{i}^{T} \boldsymbol{e}\right)\left(\boldsymbol{e}_{a}^{T} \boldsymbol{e}_{j}\right)=0 .
$$

(A proof can be constructed via determinants and cofactors defining the inverses $G_{a a}^{(c)}$ and $G_{a a}^{(r)}$ upon noting that in constructing $I-P+\boldsymbol{e}_{a} \boldsymbol{p}_{a}^{(r) T}$ the only elements of $I-$ $P$ that are changed are in the $a$-th row where each element is zero apart from the $(a, a)$ th element which is 1 . Similarly that in constructing $I-P+\boldsymbol{p}_{a}^{(c)} \boldsymbol{e}_{a}^{T}$ the only elements of $I-P$ that are changed are in the a-th column where each element is zero apart from the (a, a)-th element which is 1.) 


\section{Mean first passage times}

Let $M=\left[m_{i j}\right]$ be the mean first passage time matrix of a finite irreducible Markov chain with transition matrix $P$. All known general procedures for finding mean first passage times involve the determination of either matrix inverses or g-inverses. The following theorem summarises the general determination of $M$ by solving the well known equations for the $m_{i j}$ :

$$
m_{i j}=1+\sum_{k \neq j} p_{i k} m_{k j}
$$

using g-inverses to solve the matrix equation $(I-P) M=E-P M_{d}$, where $E=\boldsymbol{e} e^{T}=$ [1] and $D=M_{d}=\left(\Pi_{d}\right)^{-1}$ with $\Pi=\boldsymbol{e} \pi^{T}$.

\section{Theorem 4.1:}

(a) Let $G$ be any g-inverse of $I-P$, then

$$
M=\left[G \Pi-E(G \Pi)_{d}+I-G+E G_{d}\right] D .
$$

(b) Let $H=G(I-\Pi)$, then

$$
M=\left[E H_{d}-H+I\right] D .
$$

(c) Let $C=I-H$, then

\section{Proof:}

$$
M=\left[C-E C_{d}+E\right] D .
$$

(a) Expression (4.2) appears in Hunter [3] as Theorem 7.3.6 having initially appeared in the literature in Hunter [2].

(b) Expression (4.3) follows from (4.2) upon substitution. The technique was also used in a disguised form in Corollary 3.1.1 of Hunter [6].

(c) Expression (4.4) follows from (4.3). It was first derived in Hunter [6].

The advantages of expressions (4.3) and (4.4) is that we can deduce simple elemental forms of $m_{i j}$ direct from these results.

Corollary 4.1.1: Let $G=\left[\mathrm{g}_{\mathrm{ij}}\right], H=\left[h_{i j}\right]$, and $C=\left[c_{i j}\right]$ then

$$
m_{i j}=\left[c_{i j}-c_{i j}+1\right] \frac{1}{\pi_{j}}, \text { for all } i, j \text {. }
$$

(b)

$$
m_{i j}=\left[h_{j j}-h_{i j}+\delta_{i j}\right] \frac{1}{\pi_{j}}= \begin{cases}\frac{1}{\pi_{j}}, & i=j, \\ {\left[h_{j j}-h_{i j}\right] \frac{1}{\pi_{j}},} & i \neq j .\end{cases}
$$

(c)

$$
m_{i j}=\left[g_{j j}-g_{i j}+\delta_{i j}\right] \frac{1}{\pi_{j}}+\left[g_{i .}-g_{j}\right], \text { for all } i, j \text {. }
$$




\section{Proof:}

(a) Result (4.5) follows directly from (4.4) (correcting the results given in Hunter [6]).

(b) Result (4.6) follows either from (4.3) or (4.5) since $h_{i j}=\delta_{i j}-c_{i j}$.

(c) Since $H=G-G \Pi$,

$$
h_{i j}=g_{i j}-\sum_{k=1}^{m} g_{i k} \pi_{j}=g_{i j}-g_{i .} \pi_{\mathrm{j}}, \text { for all } i, j .
$$

and result (4.7) follows from (4.6). Note also that since $C=I-H$

$$
c_{i j}=\delta_{i j}-g_{i j}+\sum_{k=1}^{m} g_{i k} \pi_{j}=\delta_{i j}-g_{i j}+g_{i} \pi_{j} \text {, for all } i, j \text {. }
$$

and hence result (4.7) follows alternatively from (4.5).

Note that expression (4.5) has the advantage that no special treatment of the $i=j$ case is required.

The following joint computation procedure for $\pi_{j}$ and $m_{i j}$ was given in Hunter [6], based upon Theorem 3.4 and Corollary 4.1.1(c) above. (The version below corrects some minor errors given in the initial derivation.)

\section{Theorem 4.2:}

1. Compute $G=\left[g_{i j}\right]$, be any g-inverse of $I-P$.

2. Compute sequentially rows $1,2, \ldots r(\leq m)$ of $A=I-(I-P) G \equiv\left[a_{i j}\right]$ until

$$
\sum_{k=1}^{m} a_{r k}
$$

$(1 \leq r \leq m)$ is the first non-zero sum.

3. Compute $\pi_{j}=\frac{a_{r j}}{\sum_{k=1}^{m} a_{r k}}, j=1, \ldots, m$.

4. Compute $m_{i j}= \begin{cases}\frac{\sum_{k=1}^{m} a_{r k}}{a_{r j}}, & i=j, \\ \frac{\left(g_{j j}-g_{i j}\right) \sum_{k=1}^{m} a_{r k}}{a_{r j}}+\sum_{k=1}^{m}\left(g_{i k}-g_{j k}\right), & i \neq j .\end{cases}$

While this theorem outlines a procedure for the joint computation of all the $\pi_{j}$ and $m_{i j}$ following the computation of any g-inverse, the procedure contains the unnecessary additional computation of the elements of $A$.

Observe also that all the expressions of Corollary 4.1.1 require knowledge of the stationary probabilities $\pi_{j}$. We consider instead first deriving expressions for $m_{i j} \pi_{j}$. Let $N$ $=\left[n_{i j}\right]=\left[\left(1-\delta_{i j}\right) m_{i j} \pi_{j}\right]$ so that $N=\left(M-M_{d}\right)\left(M_{d}\right)^{-1}$. Note that $n_{j j}=0$ for all $j$.

Theorem 4.3 follows directly from (4.3) and (4.4), or by solving the matrix equation $(I-P) N=\Pi-I$, using g-inverse techniques. 
Theorem 4.3: $N=\left[n_{i j}\right]=\mathrm{E} H_{d}-H$ where $H=G(I-\Pi)$, so that

$$
n_{i j}=\left(g_{j j}-g_{i j}\right)+\left(g_{i .}-g_{j}\right) \pi_{j}, \text { for all } i, j \text {. }
$$

Further, $\quad m_{i j}=\left\{\begin{array}{cc}1 / \pi_{j}, & i=j, \\ \left(g_{j j}-g_{i j}\right) / \pi_{j}+\left(g_{i}-g_{j}\right), & i \neq j .\end{array}\right.$

Let us consider using the special g-inverses given in Table 1 and 2 to find expressions for all the $\pi_{j}$ and the $m_{i j}$. The results are summarised in Table 3 .

Note that simplification of the expressions for $m_{i j}$ using $G_{e e}, G_{e b}^{(r)}$ and $G_{e b}$ results from the observation that $\boldsymbol{g}_{\text {rowsum }}$ is in each case constant. The special case of $G_{e b}$ deserves highlighting.

Theorem 4.4: If $G_{e b}=\left[I-P+e \boldsymbol{e}_{b}^{T}\right]^{-1}=\left[g_{i j}\right]$, then $\pi_{j}=g_{b j}, \quad j=1,2, \ldots, m$,

and

$$
m_{i j}= \begin{cases}1 / g_{b j}, & i=j, \\ \left(g_{j j}-g_{i j}\right) / g_{b j}, & i \neq j .\end{cases}
$$

This is one of the simplest computational expressions for both the stationary probabilities and the mean first passage times for a finite irreducible Markov chain. These results do not appear to have been given any special attention in the literature.

If the stationary probability vector has already been computed then the standard procedure is to compute either Kemeny and Snell's 'fundamental matrix', ([7]), $Z \equiv[I-$ $P+\Pi]^{-1}$, where $\Pi=\boldsymbol{e} \pi^{\prime}$, or Meyer's 'group inverse', ([8]), $A^{\#} \equiv Z-\Pi$. Both of these matrices are in fact g-inverses of $I-P$. The relevant results, which follow from Corollary 4.1.1 (c) are as follows.

\section{Theorem 4.5:}

(a) If $Z=\left[I-P+\boldsymbol{e} \pi^{T}\right]^{-1}=\left[z_{i j}\right]$ then $M=\left[m_{i j}\right]=\left[I-Z+E Z_{d}\right] D$, and

$$
m_{i j}= \begin{cases}1 / \pi_{j}, & i=j ; \\ \left(z_{j j}-z_{i j}\right) / \pi_{j}, & i \neq j .\end{cases}
$$

(b) If $A^{\#}=\left[I-P+\boldsymbol{e} \pi^{T}\right]^{-1}-\boldsymbol{e} \pi^{T}=\left[a_{i j}^{\#}\right]$ then $M=\left[m_{i j}\right]=\left[I-A^{\#}+E A_{d}^{\#}\right] D$ and

$$
m_{i j}= \begin{cases}1 / \pi_{j}, & i=j \\ \left(a_{j j}^{\#}-a_{i j}^{\#}\right) / \pi_{j}, & i \neq j .\end{cases}
$$

Proof: See Hunter, [3], Corollary 7.3.6C. These are also special cases of (4.5) since Ze $=\mathbf{e}$ and $A^{\#} \mathbf{e}=\mathbf{0}$, so that $\Sigma_{j} z_{i j}=z_{i .}=1$ for all $i$ and $\Sigma_{j} a_{i j}^{\#}=a_{i .}^{\#}=0$ for all $i$. 
Note the similarity between the expressions (4.9), (4.10) and (4.11), with (4.9) obviously the easiest of the three expressions to compute.

Table 3: Joint computation of $\left\{\pi_{j}\right\}$ and $\left[m_{i j}\right]$ using special g-inverses

\begin{tabular}{|c|c|c|c|}
\hline g-inverse & $\pi_{j}$ & $m_{i j}$ & $m_{i j}(i \neq j)$ \\
\hline$G_{e e}$ & $g_{. j}$ & $1 / g_{. j}$ & $\left(g_{j j}-g_{i j}\right) / g_{. j}$ \\
\hline$G_{e b}^{(r)}$ & $\sum_{k} p_{b k} g_{k j}$ & $1 / \sum_{k} p_{b k} g_{k j}$ & $\left(g_{j j}-g_{i j}\right) / \sum_{k} p_{b k} g_{k j}$ \\
\hline$G_{e b}$ & $g_{b j}$ & $1 / g_{b j}$ & $\left(g_{j j}-g_{i j}\right) / g_{b j}$ \\
\hline$G_{a e}^{(c)}$ & $g_{. j} / g_{. .}$ & $g_{. .} / g$. & $\left(g_{j j}-g_{i j}\right) g_{. .} / g_{.}+\left(g_{i .}-g_{j}\right)$ \\
\hline$G_{a b}^{(c, r)}$ & $\frac{\sum_{k} p_{b k} g_{k j}}{\sum_{i} \sum_{s} p_{b i} g_{i s}}$ & $\frac{\sum_{i} \sum_{s} p_{b i} g_{i s}}{\sum_{k} p_{b k} g_{k j}}$ & $\frac{\left(g_{j j}-g_{i j}\right) \sum_{i} \sum_{s} p_{b i} g_{i s}}{\sum_{k} p_{b k} g_{k j}}+\left(g_{i .}-g_{j}\right)$ \\
\hline$G_{a b}^{(c)}$ & $g_{b j} / g_{b .}$ & $g_{b .} / g_{b j}$ & $\left(g_{j j}-g_{i j}\right) g_{b .} / g_{b j}+\left(g_{i .}-g_{j .}\right)$ \\
\hline$G_{a e}$ & $g_{. j} / g_{. .}$ & g.. $/ g$. & $\left(g_{j j}-g_{i j}\right) g_{. .} / g+\left(g_{i .}-g_{j .}\right)$ \\
\hline$G_{a b}^{(r)}$ & $\frac{\sum_{k} p_{b k} g_{k j}}{\sum_{i} \sum_{s} p_{b i} g_{i s}}$ & $\frac{\sum_{i} \sum_{s} p_{b i} g_{i s}}{\sum_{k} p_{b k} g_{k j}}$ & $\frac{\left(g_{j j}-g_{i j}\right) \sum_{i} \sum_{s} p_{b i} g_{i s}}{\sum_{k} p_{b k} g_{k j}}+\left(g_{i .}-g_{j}\right)$ \\
\hline$G_{a b}$ & $g_{b j} / g_{b}$ & $g_{b .} / g_{b j}$ & $\left(g_{j j}-g_{i j}\right) g_{b .} / g_{b j}+\left(g_{i .}-g_{j .}\right)$ \\
\hline$G_{t b}^{(c)}$ & $g_{b j}$ & $1 / g_{b j}$ & $\left(g_{j j}-g_{i j}\right) / g_{b j}+\left(\delta_{i b}-\delta_{b j .}\right)$ \\
\hline
\end{tabular}

If $G=G_{t b}^{(c)}=\left[g_{i j}\right]$ then $m_{i j}=\frac{g_{j j}-g_{i j}+\delta_{i j}}{g_{b j}}+\delta_{b i}-\delta_{b j}$, the elemental expressions of $M$, as given by Corollary 7.3.6D(b) of Hunter, [3]. It also appears, in the case $b=m$, in Meyer [9].

We have been exploring structural results. If one wished to find a computationally efficient algorithm for finding $\pi_{j}$ based upon $G_{e b}$ note that for $\pi^{T}$ we need to solve the equations $\pi^{T}=\pi^{T} P$, or $\pi^{T}\left(I-P+e \boldsymbol{e}_{b}^{T}\right)=\boldsymbol{e}_{b}^{T}$. This reduces the problem to finding an efficient package for solving this system of linear equations. Paige, Styan and Wachter [10] recommended solving for $\pi$ using $\pi^{T}\left(I-P+\boldsymbol{e} \boldsymbol{u}^{T}\right)=\boldsymbol{u}^{T}$ with $\boldsymbol{u}^{T}=\boldsymbol{e}_{j}^{T} P=\boldsymbol{p}_{j}^{(r) T}$, using Gaussian elimination with pivoting. Other suggested choices included $\boldsymbol{u}^{T}=\boldsymbol{e}_{j}^{T}$, the recommended algorithm above. We do not explore such computational procedures in this paper. It is however interesting to observe that the particular matrix inverse we suggest has been proposed in the past as the basis for a computational procedure for solving for the stationary probabilities. Mean first passage times were not considered in 
[9] and techniques for finding the $m_{i j}$ typically require the computation of a matrix inverse. $G_{e b}$ appears to be a suitable candidate.

In deriving the mean first passage times one is in effect solving the set of equations (4.1). If in this set of equations if we hold $j$ fixed, $(j=1,2, \ldots, m)$ and let $\boldsymbol{m}_{j}^{T}=\left(m_{1 j}\right.$, $\left.m_{2 j}, \ldots, m_{m j}\right)$ then equation (4.1) yields

$$
\boldsymbol{m}_{j}=\left[I-P+\boldsymbol{p}_{j}^{(c)} \boldsymbol{e}_{j}\right]^{-1} \boldsymbol{e}=G_{j j}^{(c)} \boldsymbol{e} .
$$

(This result appears in Hunter [3], as Corollary 7.3.3A). Note the appearance of one of the special g-inverses considered in this paper of the form of $G_{a a}^{(c)}$ with $a=j$.

Theorem 4.6: For fixed $j, 1 \leq i \leq m$,

$$
m_{i j}=\boldsymbol{e}_{i}^{T} G_{j j}^{(c)} \boldsymbol{e}
$$

Further, if $G_{j j}^{(c)}=\left[g_{r s}\right]$ then $m_{i j}=g_{i \text {. }}$

$$
m_{i j}=\boldsymbol{e}_{i}^{T} G_{j j}^{(r)} \boldsymbol{e}+\frac{\delta_{i j}}{\pi_{j}}-1 .
$$

Further, if $G_{j j}^{(r)}=\left[g_{r s}\right]$ then $m_{i j}=g_{i .}+\frac{\delta_{i j}}{\pi_{j}}-1=\left\{\begin{array}{cc}\sum_{k=1}^{m} p_{j k} g_{k .}, & i=j, \\ g_{i .}-1, & i \neq j .\end{array}\right.$

$$
m_{i j}=\boldsymbol{e}_{i}^{T} G_{j j} \boldsymbol{e}+\frac{\delta_{i j}-1}{\pi_{j}}
$$

Further, if $G_{j j}=\left[g_{r s}\right]$ then $m_{i j}= \begin{cases}g_{j} . & i=j, \\ g_{i .}-g_{j} . & i \neq j .\end{cases}$

\section{Proof:}

Expressions (4.13), (4.14), and (4.15) follow, respectively, from (4.12), (3.19) and (4.13), and (3.20) and (4.14) (or (3.21) and (4.13)). The elemental expressions for $m_{i j}$ follow as the $i$-th component of the $\boldsymbol{g}_{\text {rowsum }}$ of $G_{j j}^{(c)}, G_{j j}^{(r)}$, and $G_{j j}$. For case (b), from Table 2 it follows that $g_{j .}=1$ and $\sum_{k=1}^{m} p_{j k} g_{k}=\boldsymbol{p}_{j}^{(r) T} G_{j j}^{(r)} \boldsymbol{e}=1 / \pi_{j}$. For case (c) observe that $g_{j .}=\boldsymbol{g}_{j}^{(r) T} \boldsymbol{e}=1 / \pi_{j}$.

All of these results are consistent with equation (4.8). For example, for (4.12), with $G_{j j}^{(c)}=\left[g_{i j}\right]$, from equation (3.14), $\pi_{i}=g_{j i} / g_{j}$ for all $i$. Observe that from Table 2 that the $j$-th row and column of $G_{j j}^{(c)}$ are, respectively, $\pi^{T} / \pi_{j}$ and $\boldsymbol{e}_{j}$, so that for fixed $j, g_{j j}=1$, and for $i \neq j$, $g_{i j}=0$ and $g_{j i}=\pi_{i} / \pi_{j}$ with $g_{j}=1 / \pi_{j}$. Substitution in (4.7), for fixed $j$, yields $m_{j j}=1 / \pi_{j}=g_{j .}$ and for $i \neq j, m_{i j}=\left(g_{j j}-g_{i j}\right) g_{j}+\left(g_{i .}-g_{j}\right)=g_{i}$, as given by (4.13). 
The utilisation of special matrix inverses as g-inverses in the joint computation of stationary distributions and mean first passage times leads to a significant simplification in that at most a single matrix inverse needs to be computed and often this involves a row or column sum with a very simple form, further reducing the necessary computations. While no computational examples have been included in this paper, a variety of new procedures have been presented that warrant further examination from a computational efficiency perspective.

\section{References}

1. Decell, H.P., Jr., and Odell P. L. (1967). On the fixed point probability vector of regular or ergodic transition matrices. Journal of the American Statistical Association, 62, 600-602.

2. Hunter J.J. (1982). Generalized inverses and their application to applied probability problems. Linear Algebra Appl., 45, 157-198.

3. Hunter, J.J. (1983). Mathematical Techniques of Applied Probability, Volume 2, Discrete Time Models: Techniques and Applications. Academic, New York.

4. Hunter J.J. (1988). Charaterisations of generalized inverses associated with Markovian kernels. Linear Algebra Appl., 102, 121-142.

5. Hunter J.J. (1990). Parametric forms for generalized inverses of Markovian kernels and their applications. Linear Algebra Appl., 127, 71-84.

6. Hunter J.J. (1992). Stationary distributions and mean first passage times in Markov chains using generalized inverses. Asia-Pacific Journal of Operational Research, 9, 145-153.

7. Kemeny J.G. and Snell, J.L. (1960). Finite Markov Chains. Van Nostrand, New York.

8. Meyer C.D. Jr. (1975). The role of the group generalized inverse in the theory of finite Markov chains. SIAM Rev., 17, 443-464.

9. Meyer C.D. Jr. (1978). An alternative expression for the mean first passage time matrix. Linear Algebra Appl., 22, 41-47.

10. Paige C.C., Styan G.P.H., and Wachter P.G. (1975). Computation of the stationary distribution of a Markov chain. J. Statist. Comput. Simulation, 4, 173-186. 